\title{
Genetic variation in caspase-1 as predictor of accelerated progression from mild cognitive impairment to Alzheimer's disease
}

Ana Pozueta, José Luis Vázquez-Higuera, Pascual Sánchez-Juan, Eloy Rodríguez-Rodríguez, Coro Sánchez-Quintana, Ignacio Mateo, José Berciano, Onofre Combarros

Neurology Service and CIBERNED, “Marqués de Valdecilla” University Hospital (University of Cantabria and IFIMAV), Santander, Spain

Key words: Alzheimer's disease, Early detection, Mild cognitive impairment, APOE, Caspase-1, Genetic polymorphism

Correspondence to: Onofre Combarros, Neurology Service, University Hospital "Marqués de Valdecilla” (University of Cantabria), 39008-Santander, Spain. Tel. 34-942-202507; Fax: 34942-202655; E-mail: combarro@unican.es 
Sirs: There has been a lot of interest on the detection of predictors of conversion from mild cognitive impairment (MCI) to Alzheimer's disease (AD) using neuroimaging methods, CSF biomarkers and cognitive tests [2]. Despite the many genetic studies of $\mathrm{AD}$, there has been little research directed toward determining the influence of genetic variation on progression from MCI to AD $[1,4]$. Variation in the caspase-1 (CASP1) gene has been evaluated in both cognitive function in elderly individuals with normal cognition [5] and in predisposition to $\mathrm{AD}[6]$, but no previous study has specifically explored the association between genetic variation in CASP1 and progression from MCI to AD. As a primary goal in this study, we investigate APOE $\varepsilon 4$ allele and CASP1 (rs580253) polymorphisms in order to predict MCI individuals likely to convert to AD in a relatively short follow-up period.

Between 2007 and 2009, we examined 84 consecutive patients who attended the Department of Neurology of University Hospital “Marqués de Valdecilla” (Santander, Spain) and that fulfilled the Petersen criteria for amnestic MCI [3]. Patients with MCI were longitudinally assessed at 6-month intervals (mean duration of follow-up 26.5 months; SD 8.1; range 7-37 months); except one MCI-converter patient with a minimal follow-up period of 7 months, the rest of our patients were followed up for at least one year to avoid the risk to label as MCI-nonconverter subjects who will develop dementia over time. According to their Clinical Dementia Rating (CDR) scores during the follow-up period, 50 MCI patients whose global CDR score changed from $\mathrm{CDR}=0.5$ to $\mathrm{CDR}=1$ were diagnosed as MCI-converters to $\mathrm{AD}$ (70\% women; mean age 76.3 years; SD 5.7; range 60-85 years), and two additional MCI patients converted to dementia with Lewy bodies and vascular dementia, respectively; 32 MCI whose global CDR score remained stable were classified as MCI-nonconverters to AD (63\% women; mean age 74.8 years; SD 7.0; range 60-86 years). We selected the CASP1 gene (rs580253) polymorphism that had been previously related to cognitive function [5]. Blood samples were taken after written informed consent had been obtained from the subjects or 
their representatives. The study was approved by the ethical committee of the University Hospital "Marqués de Valdecilla”. Kaplan Meier curves and Cox regression model (including APOE status, age and gender) were used to assess whether time-to-conversion was associated with CASP1 (rs580253) polymorphism.

Kaplan-Meier survival analysis (Figure 1), shows the effect of APOE $\varepsilon 4$ allele and CASP1 (rs580253) T allele on the time-to-conversion to AD in patients with MCI. Survival function estimates did not differ significantly between APOE $\varepsilon 4$ allele carriers and noncarriers. Conversely, the mean time of conversion from MCI to AD was 25.2 months (SD 9.1) in people with CASP1 (rs580253) T allele (CT and TT genotypes) versus 31.7 months (SD 7.4) in CASP1 (rs580253) T allele noncarriers (CC genotype); thus, CASP1 (rs580253) T allele accelerated the progression from MCI to AD by an average of 6.5 months $(\mathrm{P}=0.009)$. The risk factors of conversion from MCI to AD were analyzed simultaneously with a multivariate Cox regression model: CASP1 (rs580253) T allele was significantly associated with faster progression from MCI to AD (Hazard Ratio = 1.88, 95\% CI 1.02-3.50, $\mathrm{P}=0.04$ ), whereas age $(\mathrm{HR}=0.98,95 \%$ CI 0.93-1.05, $\mathrm{P}=0.66)$, gender $(\mathrm{HR}=1.29,95 \%$ CI 0.67-2.49, $\mathrm{P}=0.44)$, and APOE $\varepsilon 4$ allele $(\mathrm{HR}=1.26,95 \% \mathrm{CI} 0.67-2.38, \mathrm{P}=0.47)$ did not reach statistical significance.

The main goal of this study was to analyze the usefulness of genetic variation in APOE and CASP1 as biomarkers for progression from MCI to AD in a relatively short period (the average follow-up period in this study was 26.5 months). The APOE $\varepsilon 4$ allele did not shorten time-to-progression from MCI to AD in our series. MCI patients with the APOE $\varepsilon 4$ allele have been described to be more likely to progress to $\mathrm{AD}$ as compared to those without the APOE $\varepsilon 4$ allele [4], but these studies of AD conversion frequently do not account for timedependent progression. According with our results, Barabash et al. [1] did not find that APOE $\varepsilon 4$ allele was a predictive factor of progression from MCI to AD before 20 months of evolution, but these authors observed that Ala/Thr alpha1-antichymotripsin polymorphism 
was a significative predictor of rapid conversion from MCI to AD over this short follow-up period. Similarly, in the present study CASP1 (rs580253) T allele accelerated the progression from MCI to $\mathrm{AD}(\mathrm{HR}=1.88)$ and shortened time-to-progression by 6.5 months. In conclusion, CASP1 is a modifier gene for the time of progression from MCI to AD, in that it might identify patients with MCI who are more likely to rapidly convert to AD during a short follow-up period.

\section{References}

1. Barabash A, Marcos A, Ancín I, Vázquez-Alvarez B, de Ugarte C, Gil P, Fernández C, Encinas M, López-Ibor JJ, Cabranes JA (2009) APOE, ACT and CHRNA7 genes in the conversión from amnestic mild cognitive impairment to Alzheimer’s disease. Neurobiol Aging 30:1254-1264

2. Landau SM, Harvey D, Madison CM, Reiman EM, Foster NL, Aisen PS, Petersen RC, Shaw LM, Trojanowski JQ, Jack CR Jr, Weiner MW, Jagust WJ, Alzheimer’s Disease Neuroimaging Initiative (2010) Comparing predictors of conversion and decline in mild cognitive impairment. Neurology 75:230-238

3. Petersen RC, Doody R, Kurz A, Mohs RC, Morris JC, Rabins PV, Ritchie K, Rossor M, Thal L, Winblad B (2001) Current concepts in mild cognitive impairment. Arch Neurol 58:1985-1992

4. Reitz, C, Mayeux R (2010) Use of genetic variation as biomarkers for mild cognitive impairment and progression of mild cognitive impairment to dementia. J Alzheimers Dis 19:229-251

5. Trompet S, de Craen AJM, Slagboom P, Shepherd J, Blauw GJ, Murphy MB, Bollen ELEM, Buckley BM, Ford I, Gaw A, Macfarlane PW, Packard CJ, Stott DJ, Jukema JW, Westendorp RGJ, PROSPER Group (2008) Genetic variation in the interleukin- 
I $\beta$-converting enzyme associates with cognitive function. The PROSPER study. Brain 131:1069-1077

6. Vázquez-Higuera JL, Rodríguez-Rodríguez E, Sánchez-Juan P, Mateo I, Pozueta A, Martínez-García A, Frank A, Valdivieso F, Berciano J, Bullido MJ, Combarros O (2010) Caspase-1 genetic variation is not associated with Alzheimer's disease risk. BMC Med Genet 11:32.

Figure 1. Effect of APOE (A) and CASP1 rs580253 (B) genetic variation on survival time (Kaplan-Meier analysis) to Alzheimer's disease (AD) in patients with mild cognitive impairment (MCI). The y-axis shows the cumulative proportion of MCI-nonconverters to AD for any given follow-up period on the x-axis. 
A)

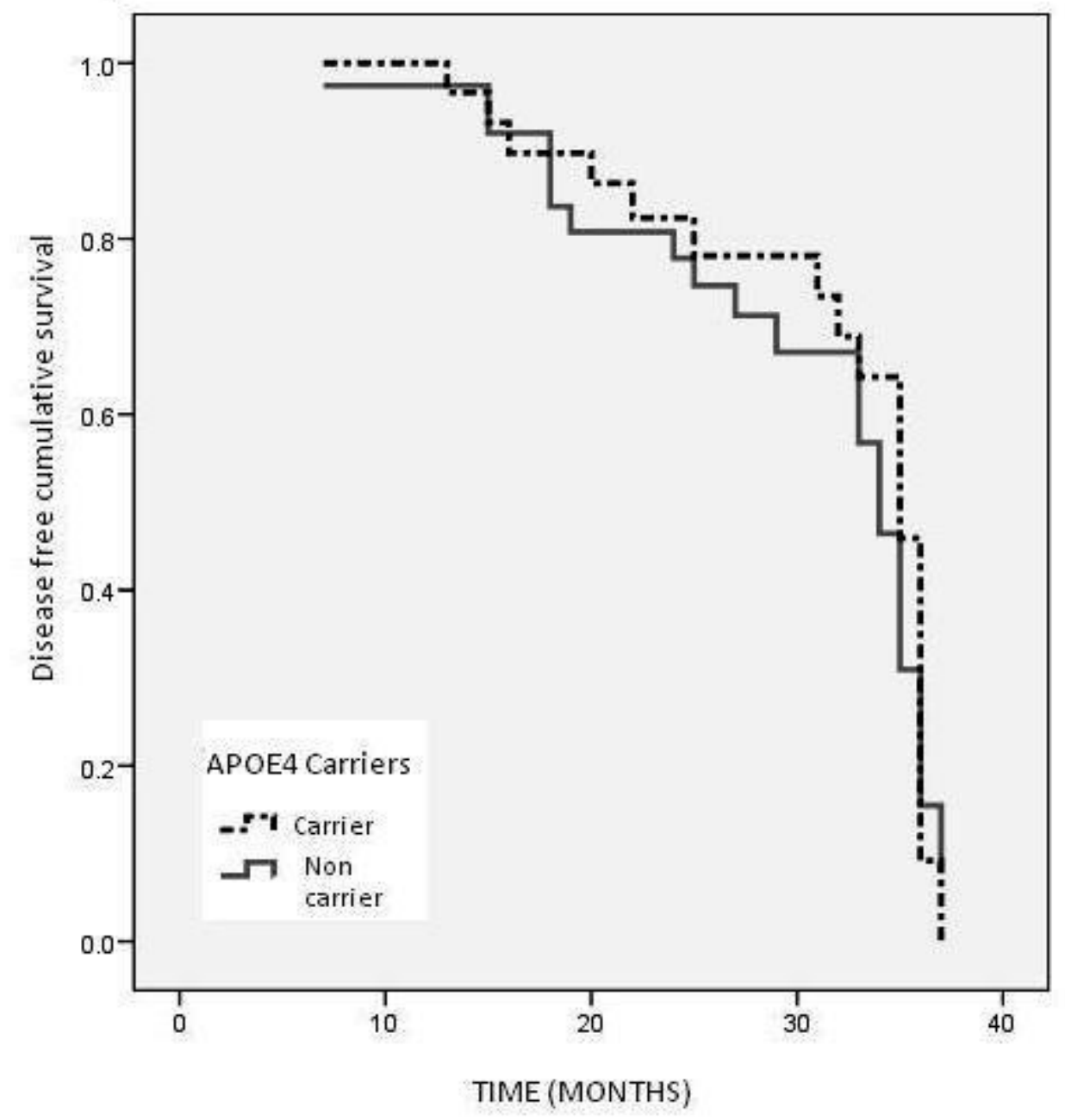

B)

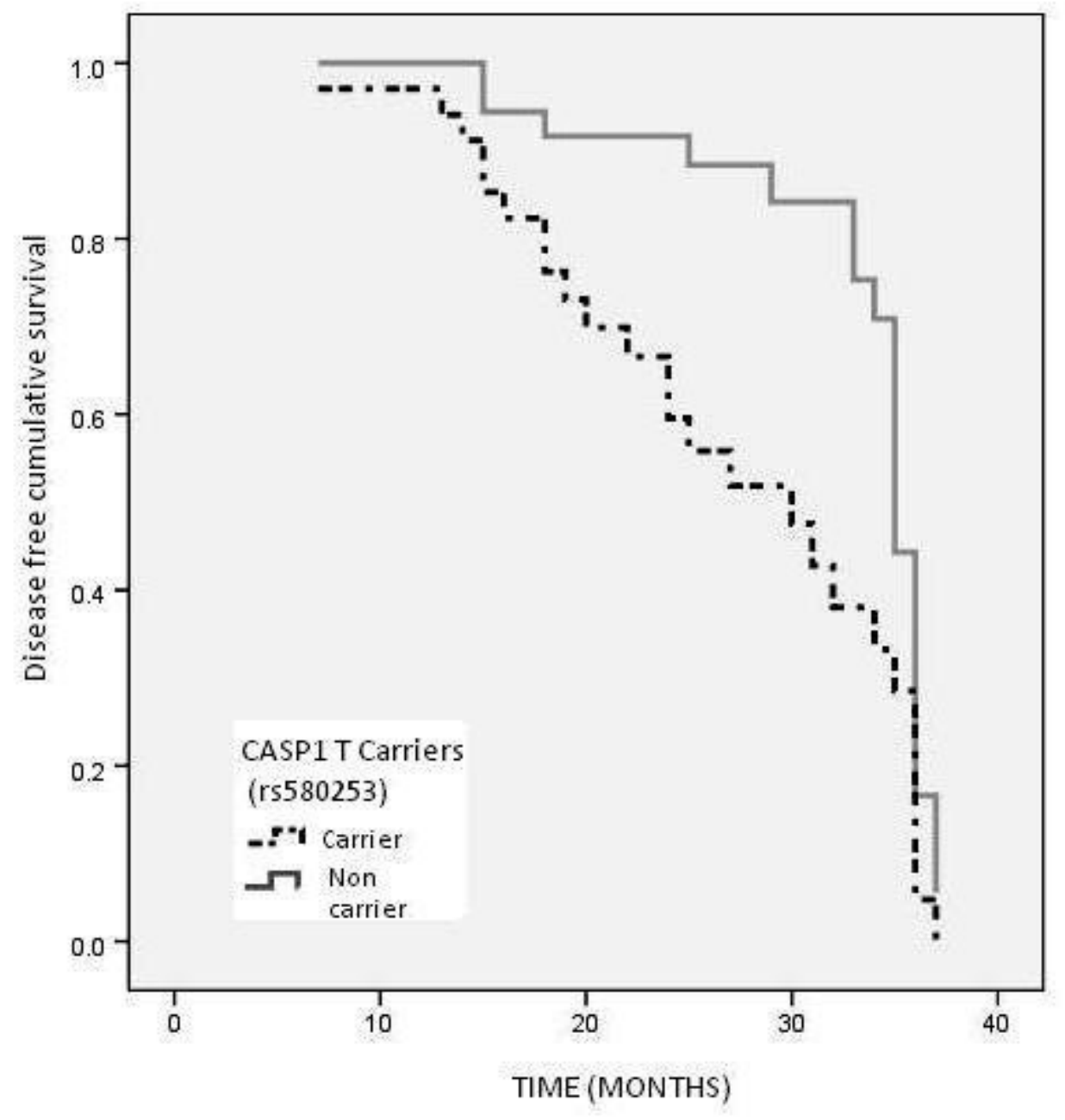

